\title{
10 ADHS und Jugendhilfe
}

Jörg Michael Fegert

\subsection{Einleitung}

In den letzten Jahren ist es immer wieder zu Auseinandersetzungen um Leistungen der Eingliederungshilfe aus der Jugendhilfe gekommen. Im Zentrum der Debatte standen dabei so genannte Teilleistungsstörungen wie Legasthenie und Dyskalkulie, wo häufig der Verdacht bestand, dass sich rechtsanwaltlich gut vertretende Mittelschichteltern, ohne dass eine tatsächliche Teilhabebeeinträchtigung ihrer Kinder besteht hier oder droht „Nachhilfe auf Staatskosten" beschaffen.

In der kinder- und jugendpsychiatrischen Praxis gibt es bei Kindern mit einer hyperkinetischen Störung, welche nach den Kriterien der ICD-1o diagnostiziert ist, weniger Probleme bei einer auf diesem Störungsbild beruhenden Teilhabebeeinträchtigung entsprechende Hilfen zu realisieren. Ganz anders scheint das Bild zu sein, spricht man mit Kinderärzten und Hausärzten, welche auch unkomplizierte ADHS behandeln. Hier erweist es sich nun als hoch problematisch, dass unter der Schirmherrschaft des Bundesgesundheitsminsiteriums, sich die Fachverbände auf Wunsch der Pädiater darauf einigten, dass sowohl DSM IV wie auch ICD-1o Diagnosen in Deutschland die Grundlage für die Feststellung/Behandlung einer ADHS darstellen können. Selbstverständlich gelten auch bei den DSM IV Kriterien die gleichen Symptome als Crundlage für die Diagnose. So werden für den kombinierten Typ sechs Unaufmerksamkeitssymptome und sechs Hyperaktivitäts- und Impulsivitätssymptome verlangt. Dennoch ist nach ICD-1o die Hyperkinetische Störung per definitionem eine Störung der Aufmerksamkeit und der Aktivität bei der 6 von 9 Unaufmerksamkeitsymptomen, 1 von 4 Impulsivitätssymptomen und 3 von 5 Hyperaktivitätssymptomen für die Diagnose gefordert werden. Zusätzlich 
können spezifische Störungsbilder mit ungünstiger Diagnose wie die Kombination mit Störung des Sozialverhaltens eigenständig verschlüsselt werden. Der große Unterschied zwischen DSM IV und ICD-1o liegt in der situationalen und symptomatologischen Pervasivität, d. h. wie umfassend sich die Symptomatik und über welche Alltagsfelder hinweg sie sich äußert. Ganz zentral ist der Unterschied in der funktionellen Beeinträchtigung. Dies ist relevant für die Einschätzung, ob eine Teilhabebeeinträchtigung resultiert. So wird in der ICD-1o Diagnose im Gegensatz zur DSM-IV Diagnose eine signifikante funktionelle Beeinträchtigung gefordert, welche unangemessen ist in Bezug auf Alter, Entwicklungsstadium und Intelligenz. Zu beobachten muss eine gewisse Beeinträchtigung in 2 oder mehr Alltagssettings sein, z. B. Schule, zu Hause, Freizeitbereich etc. Dies bedeutet, dass eine ICD-1o Diagnose einer Hyperkinetischen Störung immer eine funktionelle Beeinträchtigung mit beinhaltet, während dies eine DSM IV Diagnose a priori nicht tut. Vergleicht man in deutschen und schweizer Prävalenzstudien ICD-9/ICD-1o Diagnosen und DSM III/DSM IV Diagnosen, so fällt auf, dass in der gleichen Untersuchungspopulation 3-4 mal so viele DSM Diagnosen wie ICD-1o Diagnosen gestellt werden.

Santoshs Reanalyse der MTA Daten (Santosh et al. 2005) zeigte bei der Anwendung von 4 so genannten ICD-10 „Filtern“ (Komorbidität, Symptombereich, Pervasivität und Beeinträchtigung im Alltag), dass von ursprünglich 579 Kindern aus dem MTA Sample 145 eine hyperkinetische Störung nach ICD1o hatten. Gerade die Filter Pervasivität (also auftreten der Störung in mehreren Bereichen) und Behinderung und Beeinträchtigung führten zu einer erheblichen Reduktion. Dies hat auch Relevanz für die Leistungsgewährung in der Jugendhilfe, denn entsprechend der sonstigen Vorgehensweise im Gesundheitssystem stellt das Kinder- und Jugendhilfegesetz, SGB VIII, nun bei der Feststellung einer seelischen Behinderung direkt auf ICD-1o Diagnosen als Diagnosekriterien ab. Wörtlich heißt es im $\$ 35$ a:

„Die Stellungnahme ist auf der Crundlage der Internationalen Klassifikation der Krankheiten in der vom Deutschen Institut für Medizinische Dokumentation und Information herausgegebenen deutschen Fassung zu erstellen."

Dies bedeutet, dass auf die jeweilige im SGB V geltende ICD Klassifikation Bezug genommen werden muss.

Gerade weil sich einfache Aufmerksamkeitsstörungen nach DSM problemlos medikamentös behandeln lassen - eine Übersicht zu den Studien findet sich in Hässler und Fegert (2004) - ist es also verständlich, wenn die Jugendhilfe gar nicht in die Prüfung der zweiten Ebene der Anspruchsberechtigung eintritt, wenn auf der ersten Feststellungsebene nicht auf die vorhandenen Kriterien (ICD-1o Diagnose) Bezug genommen wird. Um hier nicht falsch verstanden zu werden: Selbstverständlich haben viele Kinder mit einer hyperkinetischen Störung eine drohende oder schon bestehende Teilhabebeeinträchtigung und benötigen deshalb Eingliederungshilfen nach dem Kinder- und 
Jugendhilfegesetz. Es gibt aber auch, vor allem bei den Kindern mit einfacher Aufmerksamkeitsstörung nicht wenige Kinder und Jugendliche, die insgesamt mit einer fachkundigen kinder- und jugendpsychiatrischen oder pädiatrischen Behandlung und Medikamenteneinstellung, allein mit dieser Unterstützung aus dem Gesundheitssystem zur Teilhabe ohne größere Einschränkungen in der Lage sind. Insofern kann am Beispiel der ADHS deutlich gezeigt werden, dass kein Automatismus zwischen dem Vorliegen einer psychiatrischen Diagnose und dem Anspruch auf Eingliederungshilfen besteht. Allerdings legt eine korrekte Diagnosestellung, welche bei den ICD-1o Kriterien, Kriterien des Zurechtkommens im Alltag mit einbezieht, eine drohende Beeinträchtigung sehr viel stärker nahe, als die rein deskriptiven und niedrigschwelligeren DSM-IV Diagnosen.

Insofern ist gerade die hyperkinetische Störung, in Abgrenzung zur allgemeineren ADHS-Diagnose, ein typisches Beispiel, weshalb bei der Leistungsgewährung in der Jugendhilfe im Einzelfall geprüft werden muss, ob die anspruchsbegründenden Voraussetzungen vorliegen. Dabei wird häufig übersehen, dass unter den schwerstbeeinträchtigten Kindern und Jugendlichen, welche $z$. B. stationäre Hilfen aus der Jugendhilfe bekommen, ein erheblicher Teil von Kindern mit hyperkinetischen Störungen, insbesondere hyperkinetischen Störungen des Sozialverhaltens, vertreten ist. Diesen, an der Schnittstelle zwischen Jugendhilfe und Jugendpsychiatrie häufig vernachlässigten und an der Schnittstelle zwischen Jugendhilfe und Jugendpsychiatrie nicht hinreichend beachteten Kindern sei hier nach einem definitorischen Überblick das Hauptaugenmerk geschenkt.

\section{2 Ärztliche Mitwirkung bei der Feststellung der Teilhabebeeinträchtigung}

\section{Ärztliche Rolle im Verfahren}

Der $\mathbb{3}$ 35a SGB VIII, Eingliederungshilfe für seelisch behinderte Kinder und Jugendliche, wurde bei der Novellierung des SGB VIII durch das KICK beibehalten, wenn auch verändert. Die Veränderungen zielen ab auf die Eingangsvoraussetzungen, insbesondere darauf, wer diese festzustellen hat. Hier wurden die fachlich geeigneten Personen zur Feststellung der psychischen Störung ( $\$ 35 \mathrm{a}$ Abs. 1 S. 1 Nr. 1 SGB VIII) präzisiert: der Facharzt für Kinder- und Jugendpsychiatrie/Psychotherapie sowie der Kinder- und Jugendlichenpsychotherapeut. Um der Versorgungsrealität Rechnung zu tragen, wurden auch Ärzte oder Psychotherapeuten als mögliche Verfasser einer Stellungnahme benannt, wenn sie über ,besondere Erfahrungen auf dem Gebiet seelischer Störungen bei Kindern und Jugendlichen“ verfügen ( $\$ 35$ a Abs. 1a S. 1 Nr. 3 SGB VIII). Neu ist auch, dass Feststeller und Leistungserbringer nicht mehr die gleichen Personen sein dürfen ( $\$ 35$ a Abs. 1a S. 4 SCB VIII), was einer in der Vergangenheit mitunter zu beobachtenden Praxis entgegenwirken soll, dass etwa im Bereich 
der Legasthenietherapie Leistungserbringer selbst - teilweise fragwürdige Gutachten erstellten, auf deren Crundlage sie dann vom Jugendamt bezahlte Therapien erbringen konnten.

Die Feststellung dessen, was Teilhabebeeinträchtigung ist und wie sie im Einzelfall am besten zu minimieren bzw. zu beheben ist, bleibt eine Aufgabe der Mitarbeiter der Jugendämter (Fegert 2004b). Der Facharzt/Psychotherapeut stellt also fest, ob die Eingangsvoraussetzung, eine psychische Störung, vorliegt, und bestätigt dies in einem Gutachten/einer Stellungnahme für das Jugendamt, welches dann die Auswirkungen dieser Störung in Bezug auf die Teilhabe prüft und etwaige Defizite und geeignete Hilfen zur Beseitigung der Defizite feststellt bzw. implementiert.

Natürlich kann sich der Facharzt/Psychotherapeut auch zum Ausmaß der Teilhabebeeinträchtigung und $\mathrm{zu}$ anderen eingliederungsrelevanten Fragen äußern, allerdings hat er dann kein Feststellungsmonopol, sondern ist eine Stimme im Konzert vieler und er muss akzeptieren, dass das Konzert - um in der Metapher zu bleiben - von der Jugendhilfe dirigiert wird (Federführung der Jugendhilfe). Die Kommission zur Zusammenarbeit zwischen Jugendhilfe und Jugendpsychiatrie der drei Fachgesellschaften für Kinder- und Jugendpsychiatrie in Deutschland, hat sich unter assoziierter Mitarbeit von Dr. Thomas Meysen (Fegert et al. 2008) zu Begutachtungen und zum Rollenverständnis der Beteiligten geäußert. Dabei wird klar festgehalten, dass der Störungsund Krankheitsbegriff der in der Kinder- und Jugendhilfe angewandt wird, per definitionem nun derselbe wie in der Medizin ist und dass ein einheitliches, gültiges Klassifikationssystem, derzeit die ICD-1o der WHO, angewendet wird. Dies war eine der zentralen Forderungen der Kinder- und Jugendpsychiater nach der Einführung des KJHG gewesen (vgl. Fegert in Fegert \& Schrapper 2004). Zum Verständnis ist wichtig, dass es sich bei diesen Diagnosen um einen kategorialen Krankheitsbegriff handelt. Deshalb ist es fachlich unerklärlich wenn der Gesetzgeber mit Bezug auf frühere Debatten um die Verhaltensauffälligkeiten (vor Einführung des KJHG) nun wieder politischem Druck folgend die Feststellung verlangt, dass die Störung Krankheitswert habe. Es handelt sich bei den ICD-1o Diagnosen ohnehin nicht um dimensionale Feststellungen, z. B. von Ängstlichkeit unterschiedlichen Ausmaßes, Traurigkeit etc., sondern in der Diagnosevergabe ist schon ein Schwellenwert, wie er von der WHO festgelegt ist, mit eingeschlossen (siehe oben). Es wäre insofern deutlich sinnvoller gewesen, wenn der Gesetzgeber hier Ärzte oder psychologische Psychotherapeuten, bzw. Kinder- und JugendlichenPsychotherapeuten, aufgefordert hätte, zu prüfen, welche Gesundheitsauswirkungen die Erkrankung hat und inwieweit vonseiten des Gesundheitssystems Behandlungsmöglichkeiten und Therapiemöglichkeiten bestehen oder Rehabilitations-Angebote gemacht werden könnten. Das formelhafte Herbeten, dass eine Krankheit eine Krankheit ist, weil eben die Krankheitsdefinition erfüllt ist, wird den Ärzten zwar nicht schwer fallen, ist aber für jeden, der die lange Vorgeschichte nicht kennt, aufgrund der jetzigen Vorgaben in der Norm nicht nachvollziehbar. 
Früher ist es häufig vorgekommen, dass das Jugendamt darauf bestanden hat, dass die ärztliche Stellungnahme bzw. das Gutachten zu $\$ 35$ a vom Amtsarzt erstellt wird. Im Kontext des $\mathbb{5} 35$ a SGB VIII gibt es kein Amtsarztgebot. Im Gegenteil: Das Gesetz benennt jetzt sehr detailliert, wer zur Feststellung der Eingangsvoraussetzung befähigt ist (siehe weiter oben). In vielen kleineren Gesundheitsämtern finden sich eben solche, primär genannten Fachärzte, nicht. Insofern ist eine Begutachtung durch einen fachlich geeigneten Experten vorzuziehen. Wegen der Probleme mit der Rolle des diagnostizierenden Arztes ergeben sich wiederholt auch Streitigkeiten mit den Jugendämtern um die Bezahlung von Stellungnahmen. Hierbei ist unsere Auffassung, dass im Rahmen sozialpsychiatrischer Tätigkeiten, wenn sich die Patienten selbst zur Diagnostik präsentieren, fast alle wesentlichen Kosten von der Krankenkasse getragen werden und nur noch für die Erstellung der schriftlichen Stellungnahme/des Gutachtens anfallen. Wird eine Familie hingegen vom Jugendamt mit einem expliziten Gutachtenauftrag geschickt, d. h. liegt also ein schriftlicher Auftrag zur Einholung eines schriftlichen Gutachtens vor, ohne dass eine Arzt-Patienten-Beziehung besteht, dann handelt der angegangene Arzt, bzw. der angegangene Kinder- und Jugendlichenpsychotherapeut im Auftrag des Jugendamtes und sollte entsprechend dem Justizvergütungs- und Entschädigungsgesetz (JVEG) nach dem dort angegebenen Satz für einfache gutachterliche Beurteilungen ( $\mathrm{M} 1$, derzeit $50 €$ /Stunde) abrechnen. An dieser Stelle wird im Gesetz explizit auf das Fallbeispiel eines Gutachtens der Verlängerung einer Betreuung oder nach $\$ 35$ a KJHG hingewiesen. Mancherorts wurden auch direkte Absprachen mit Jugendämtern getroffen, welche die Vergütung der ärztlichen Mitwirkung regeln.

\section{Teilhabebeeinträchtigung}

Während für körperliche Behinderungen durch die WHO seit Langem der Begriff der Teilhabe definiert und mit Inhalt gefüllt wurde, u. a. durch die International Classification of Functioning (ICF), blieb er für den Bereich der psychisch erkrankten Minderjährigen eher unscharf. Hier mangelt es an Operationalisierungen und Standardisierungen (Fegert 2004a). Dabei besteht gerade für diesen Bereich eine eigene gesetzliche Grundlage für die Teilhabe: der $\$ 35$ a SGB VIII. Dieser sieht für Kinder und Jugendliche, die durch eine psychische Erkrankung in der Teilhabe beeinträchtigt sind, explizit Eingliederungsleistungen vor. Bei der Novellierung des SGB VIII durch das Gesetz zur Weiterentwicklung der Kinder- und Jugendhilfe (KICK) ${ }^{4}$ wurde von einer genaueren Definition des Begriffs der Teilhabebeeinträchtigung abgesehen. Dabei wurde und wird vor dem Hintergrund der Kostenfolgen dieses Paragraphen gestritten, welche Anforderungen an die Teilhabebeeinträchtigung nach $\$ 35 \mathrm{a}$ Abs. 1 S. 1 Nr. 2 SGB VIII erfüllt sein müssen, um eine Leistungspflicht auszulösen.

4 Gesetz v. 8. September 2005, BGBI I, S. 2729. 
Zum Beispiel werden zur Teilhabebeeinträchtigung im Bereich der Schulkarriere mögliche Nachteile bei der Suche nach einem Arbeitsplatz in der Zukunft diskutiert und gefragt, ob dies im Sinne eines gesellschaftlichen Nachteils bereits die Tatbestandsvoraussetzung erfüllt (Wiesner et al. 2005).

Mit dem KICK wurde keine explizite Beschreibung ins Gesetz aufgenommen, wie das Feststellungsverfahren auszusehen hat bzw. welchen Standards es folgen sollte. Diese originäre Aufgabe der Jugendämter, die Feststellung der Teilhabebeeinträchtigung, ist aber gerade durch die Verwaltungsgerichte immer wieder kritisiert worden, $u$. a. wegen einer zu geringen Nachvollziehbarkeit und/oder mangelnden Standards bei der Erhebung des Teilhabedefizits. ${ }^{5}$ Die derzeitige Praxis wurde im Rahmen eines vom Bundesministerium für Familie, Senioren, Frauen und Jugend (BMFSFJ) geförderten Projekts von uns eruiert. Die Ergebnisse werden im Folgenden dargestellt, um Anforderungen an eine Vereinheitlichung und Lösungsmöglichkeiten zu einer größeren Einzelfallgerechtigkeit zu entwickeln.

\section{Begriffsdefinitionen von Teilhabe nach der WHO}

\section{Teilhabebeeinträchtigung nach ICF}

Da der Begriff der Teilhabebeeinträchtigung auf die Internationale Klassifikation der Funktionsfähigkeit, Behinderung und Gesundheit (ICF) zurückgeht (WHO 2004), sollte beim Versuch einer Definition der Teilhabebeeinträchtigung die dort zugrunde liegende Denkweise Berücksichtigung finden. Nach der WHO wird die Teilhabe charakterisiert und beschrieben durch die wahrnehmbare Teilhabe an Situationen des sozialen Lebens (involvement in life situations). Die WHO schlägt vor, zunächst das Ausmaß der Restriktion bei der Teilhabe zu definieren (first qualifier) und dann Faktoren wie die Bereiche, auf die sich die Beeinträchtigung bezieht, die notwendige Unterstützung und die subjektive Zufriedenheit oder Unzufriedenheit mit der Situation zu erfassen.

Die WHO unterscheidet zwischen der Ebene der Körperfunktionen und Strukturen einerseits und dem Aktivitätsniveau andererseits und trennt diese beiden behinderungsrelevanten Aspekte vom Aspekt der Teilhabe (Participation) ab. Schließlich berücksichtigt sie kontextuelle Faktoren, die im positiven Sinne als unterstützende Faktoren, Erleichterer, oder in der WHO-Sprache als Faszilitatoren, wirken können, oder aber im negativen Sinne als Barrieren und Hinderungsgründe, die eine Teilhabe verhindern.

$\mathrm{Zu}$ unterscheiden ist also bei der Beurteilung der Folgen einer Behinderung das Zurechtkommen auf der körperlichen Ebene. Ist diese Ebene beeinträch-

5 VG Sigmaringen JAmt 2005, 246 unter Verw. auf Stähler/Wimmer NZW 2002, 570, die versuchen, die gängige Definition von Teilhabe nach dem Gesetz und der WHO darzustellen und Möglichkeiten einer standardisierten Erfassung von Aspekten der Teilhabe mittels international eingeführter Instrumente aufzuzeigen. 
tigt, spricht die WHO von Impairment, dies entspricht ungefähr der ärztlichen Feststellung der psychischen Störung nach $\mathbb{\$} 35 \mathrm{a}$ Abs. 1 S. 1 Nr. 1 SGB VIII und hat noch nichts mit der Teilhabebeeinträchtigung zu tun. Die zweite Ebene des Zurechtkommens ist die individuelle Ebene der Person als Ganzes. Hier geht es um die schon nicht mehr allein ärztlich zu leistende Erfassung des Aktivitätsniveaus oder der individuellen Aktivitätseinschränkung. Dies ist eine wichtige Grundvoraussetzung, um überhaupt über Teilhabe oder Teilhabebeeinträchtigung nachdenken zu können, da unterschiedliche Personen in unterschiedlichen Entwicklungsphasen verschiedene Aktivitätsansprüche haben und Einschränkungen wie Barrieren nur erlebt werden, wenn die Aktivität dazu führt, dass sie wahrgenommen werden.

\section{Bereiche der Teilhabe}

Unbestritten ist, dass eine Teilhabe bei Minderjährigen altersabhängig verschiedene Lebensbereiche berührt. Je älter ein Minderjähriger ist, desto mehr werden extrafamiliäre Bereiche bei der Beurteilung von Bedeutung sein. Zum Beispiel muss beachtet werden, ob ein Jugendlicher am sozialen Leben Teilhabe besitzt, während bei kleineren Kindern Teilhabe am sozialen Leben in und über die Familie stattfindet.

Generell kann in fünf Altersgruppen unterschieden werden:

- frühe Kindheit (Säuglings- und Kleinkindalter),

- Kindheit bis zum Vorschulalter (ab dem 3. bis zum 7. Lebensjahr),

- mittlere Kindheit (ab dem 7. bis zum 12. Lebensjahr),

- Jugendalter (ab dem 12. bis zum 18. Lebensjahr) und

- frühes Erwachsenenalter (ab dem 18. bis zum 21. Lebensjahr).

Wichtig ist eine Einschätzung zentraler Meilensteine der Entwicklung bzw. Entwicklungsaufgaben in den Bereichen „motorische“, „sprachliche“, „kognitive“ und „emotionale“ Entwicklung. Gleichzeitig sollte operationalisiert werden, wie man das globale Zurechtkommen im Alltag altersentsprechend, mit Bezug auf Selbstständigkeit, Familienbeziehungen, Beziehungen zu Gleichaltrigen, Freizeitverhaltens und schulischen Leistungen, einschätzen kann.

In der Kommentarliteratur zu $\mathbb{3} 35$ a SGB VIII wird Teilhabe als „die aktive und selbstbestimmte Gestaltung des gesellschaftlichen Lebens “ beschrieben. ${ }^{6}$ Auch in der Rechtsprechung wurde der Begriff mitunter in dieser Art definiert. ${ }^{7}$ Eine Beeinträchtigung oder auch ein „Integrationsrisiko“ könne „sich auf alle Lebensbereiche erstrecken. Ein zentraler Lebensbereich für Kinder und Jugendliche ist neben der Familie und dem sozialen Umfeld die Schule. " 8 Auch

6 Wiesner/Fegert, in: Wiesner, SGB VIII, § 35 a Rn. 19.

7 VG Sigmaringen JAmt 2005, 246 unter Verw. auf Stähler/Wimmer NZW 2002, 570

8 Wiesner/Fegert, in: Wiesner, SGB VIII, \35 a Rn. 19. 
Freundeskreis und Freizeitgestaltung von Kindern und Jugendlichen werden dazugezählt. ${ }^{9}$ Teilweise wird hierbei ausdrücklich auf die ICF als fachliche

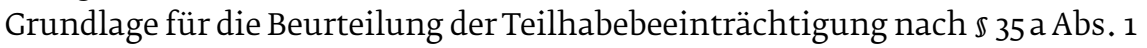
S. 1 Nr. 2 SGB VIII verwiesen. ${ }^{10}$

\subsection{Kinder mit hyperkinetischen Störungen in der stationären Jugendhilfe}

In Deutschland werden ca. 62.0oo Kinder und Jugendliche in stationärer Jugendhilfe betreut. Bei diesen Kindern handelt es sich um eine Hochrisikogruppe. Die Prävalenz psychischer Störungen liegt bei Ihnen bei über 60\%. Es dominieren externalisierende Störungen. Auffällig sind eine hohe Multimorbidität und häufig ein traumatischer Hintergrund mit oder ohne frühe Vernachlässigung. Die Weiterentwicklung und Eskalation dieser Störungsbilder ist bei diesen Kindern gekennzeichnet durch eine häufige ambulante kinderund jugendpsychiatrische Unterversorgung von Jugendhilfeeinrichtungen, durch eine Diskontinuität von Beziehungen und so genannten Drehtürkarrieren und einer zunehmend ungünstigen Kosten-/Nutzenrelation verschiedener Hilfen. Gerade wegen eines Zuständigkeitsgerangels und eines Mangels an Vernetzung zwischen Jugendhilfe und Kinder- und Jugendpsychiatrie verstärken sich durch die jetzigen Versorgungsstrukturen - teilweise ungewollt - die vorhandenen psychischen Belastungen und Störungen. Häufig liegt bei vielen Heimkindern, bei denen z. B. im Schulalter eine hyperkinetische Störung nach den ICD-1o Kriterien diagnostiziert werden kann eine entwicklungspsychopathologisch durchaus komplexere Symptomatik vor (vgl. De Bellis 20o1). Viele hyperkinetischen Symptomatiken bei diesen Kindern erweisen sich als ein entwicklungspsychopathologisches Durchgangsstadium nach früher Vernachlässigung oder als Traumafolgen von so genannten Typ 2 Traumata. Diese äußern sich zunächst in der frühen Kindheit u. a. mit:

- Bindungsstörungen, insbesondere Bindungsstörungen mit Enthemmung,

- Entwicklungsverzögerungen und

- immer stärker ausgeprägtem oppositionellem Verhalten.

In der mittleren Kindheit sind die Hauptsymptome:

- Schulversagen,

- Störungen des Sozialverhaltens,

- Dysphorie,

- Lustlosigkeit,

- Aufmerksamkeitsprobleme,

- hyperkinetische Störungen,

- depressive Symptome,

9 Münder u. a., FK-SGB VIII, 5. Aufl. 2006, \35a Rn. 33.

10 Münder u. a., FK-SGB VIII (Fn. 9), \$ 35 a Rn. 34 ff. 
- geringer Selbstwert,

- geringe soziale Kompetenz.

Im Jugendalter können dann

- Selbstverletzungen,

- Suizidalität,

- Substanzmissbrauch,

n immer stärker ausgeprägte Störungen des Sozialverhaltens,

- körperliche und sexuelle Aggressionen

hinzutreten.

Im Übergang zum Erwachsenenalter bildet sich dann bei manchen der Betroffenen eine so genannte Cluster B Persönlichkeitsstörung heraus. Manche junge Erwachsene leiden nach wie vor unter posttraumatischen Belastungssymptomen, sind suizidal, zeigen dissoziative Störungen, Abhängigkeitserkrankungen etc. und sie haben eindeutig ein erhöhtes Risiko wiederum eigene Kinder zu misshandeln. Insofern erscheint es sehr wichtig, diesen von der Vernachlässigung angestoßenen, Teufelskreis zu unterbrechen. Die Bella Studie (Ravens-Sieberer 2006) und der KIGGS-Survey (RKI 2007) zeigen, dass das relative Risiko für psychische Belastungen mit der Belastung der Familienbeziehungen ansteigt. Schon bei Alleinerziehenden besteht ein doppeltes Risiko, bei aktuellen Familienkonflikten ein fast 5-faches Risiko, bei Konflikten in der Familie der Eltern ein 2-4-faches Risiko.

Das Risiko für eine psychische Erkrankung steigt mit mehreren Belastungen und bei 3 Risiken auf ca. $30 \%$ und bei 4 Risiken auf fast $50 \%$. Das heißt, dass jedes 2. von 4 solchen Risiken betroffene Kind auch eine psychiatrische Symptomatik zeigt. Meltzer et al. (2003) fanden bei Kindern in institutioneller Betreuung über 35 \% Störungen des Sozialverhaltens und über $10 \%$ reine hyperkinetsiche Störungen. Diese Zahlen unterscheiden sich erheblich von den mit den gleichen Instrumenten ebenfalls von Meltzer et al. ermittelten Daten für die englische Allgemeinbevölkerung. Der höchste Anteil von Kindern mit ADHS fand sich bei folgenden Maßnahmen:

- sozialpädagogische Familienhilfe,

- Pflegefamilie und

- Heim.

Im betreuten Wohnen war die Diagnose deutlich seltener repräsentiert. Prädominant war in allen 4 Jugendhilfeformen die Störung des Sozialverhaltens, welche häufig auch eine hyperkinetische Störung mit Störung des Sozialverhaltens darstellt. In unserer eigenen Ulmer Heimkinderstudie (Schmid et al. 2008) konnten wir eine Gesamtstichprobe von 689 Kindern einschließen und nach einem Screening mit CBCL und YSR mit einem diagnostischen Interview nach DISYPS-KJ schließlich 359 Kinder untersuchen. Wir fanden eine Punktprävalenz für psychische Störungen von 59,9\% und mit Einschluss der Diagnose geistige Behinderung von 61,3\% bei Kindern und Jugendlichen in Jugendhilfeeinrichtungen. Fasst man hyperkinetische Störungen (F 90.0) und 
hyperkinetische Störungen des Sozialverhaltens (F go.1) zusammen, fanden wir $24 \%$ betroffene Kinder im Vergleich zu 3-6\% in Stichproben in der Allgemeinbevölkerung. Hinzu kommen noch $26 \%$ Kinder, bei denen eine Störung des Sozialverhaltens diagnostiziert werden musste, im Vergleich zu 6\% in der Allgemeinbevölkerung. Zählt man nun die Kinder mit hyperkinetischer Störung des Sozialverhaltens dazu, dann ergeben sich $26 \%+22 \%$ also $48 \%$ der Heimkinder mit einer externalisierenden Störung. Angesichts dieser massiven diagnostischen und Symtpombelastung mag der Behandlungsstatus dieser Kinder erstaunen. Nur 79, das heißt $11 \%$ der untersuchten Kinder wurden medikamentös behandelt. Methylphenidat in retardierter oder nicht retardierter Form gehörte zwar zu den am Häufigsten verordneten Medikamenten (38 Fälle). Wir fanden aber auch 18 Fälle mit Pipamperon und zahlreiche andere atypische und typische Neuroleptika in der Behandlung dieser Kinder. Vergleicht man in einer Hochrechnung aufgrund unserer Daten aus einer Expertise für das BMGS (Fegert et al. 2002), den Behandlungsstatus von Jungen mit ADHD in dem von uns angesprochenen Altersrange mit einer Ersatzkassenpopulation, welche eher mittelschichtwendig ist, so kann in der Ersatzkassenpopulation davon ausgegangen werden, dass $70-80 \%$ der Jungs mit einer entsprechenden Symptomatik im Schulalter eine medikamentöse Behandlung, insbesondere mit Methylphenidat erhalten, während bei den schwerer betroffenen Heimkindern nur um die $20 \%$ medikamentös behandelt werden. Hier scheint sich auch ein ideologisches Problem und ein Problem der Aufklärung der Bezugspersonen in pädagogischen Einrichtungen zu zeigen.

Die zweite Ulmer Heimkinderstudie war ein vom BMBF und den Krankenkassen gefördertes aufsuchendes Interventionsprogramm in Kinderheimen zur Optimierung der ambulanten Behandlungsangebote für Heimkinder, mit dem Ziel Niedrigschwelligkeit und Frühzeitigkeit von Hilfen zu erreichen. Psychosoziale Entwicklungsverläufe sollten stabilisiert werden, Jugendhilfeeinrichtungen zur langfristigen effektiven Betreuung psychisch auffälliger Jugendlicher befähigt und Drehtürkarrieren vermieden werden. Insbesondere sollten aus Sicht der Kassen Folgekosten im stationären Behandlungssektor durch rechtzeitige ambulante Behandlung vermieden werden. Bausteine des Interventionsprogramms waren ambulanten Liaisondienste, welche eine Behandlungskontinuität im Heim selbst durch ein Screening bei Aufnahme und fortlaufende Behandlung bei Bedarf sichern. Organisiert wurde diese Betreuung interdisziplinär durch Kooperation der Kinder- und Jugendpsychiatrie mit den Fachdiensten vor Ort, den Betreuern und den zuständigen Jugendämtern. Zusätzlich wurde Psychoedukation und Training für die Heimmitarbeiter angeboten, insbesondere zum Umgang mit ADHS und Störung des Sozialverhaltens. In Bezug auf die diagnostizierten Störungsbilder erfolgte eine multimodale Behandlung, leitlinienorientiert und evidenzbasiert auch unter Einsatz von Medikamenten, wo indiziert. Für aggressive und andere Krisen wurde ein zweistufiges Modell der Krisenintervention, ambulante Krisengespräche vor stationärer Aufnahme, vereinbart. Für die Jugendlichen selbst wurden spezielle störungsspezifische Interventionen, z. B. soziales Kompetenztrai- 
ning, durchgeführt. In einem Screening von 781 Jugendlichen wurden 624 eingeschlossen und auf eine Treatment as Usual-Cruppe (TAU n=336) und einer Interventionsgruppe $\mathrm{n}=\mathbf{2 8 8}$ randomisiert. Nach einem halben Jahr waren 178 Jugendliche aus der Studie, wegen Verlegung in andere Einrichtungen, Rückführung in die Familie etc. herausgefallen und nach einem weiteren halben Jahr weitere 5o. Insgesamt gelang es aber zwei hinreichend große Gruppen bis zum Ende zu untersuchen. Leider erwies sich die Interventionsgruppe als die psychosozial belastetere Gruppe. Dennoch zeigte sich eine signifikante Reduktion der stationären Behandlungstage in der Interventionsgruppe von durchschnittlich 39,8 auf 27,63 Tage. Rechnet man dies auf Behandlungstage pro Person in 12 Monaten um, so war dies ein Unterschied von 2,47 auf 1,3. Dieser Unterschied war hoch signifikant $\mathrm{P}=.0,005$, chi $^{2}=7,79$. Wir betrachteten auch, welches die Hochrisikoprobanden sind, welche aus der Heimbetreuung herausfielen und fanden hier, dass Jugendliche mit dissozialem und aggressivem Verhalten hoch signifikant häufiger zu den Abbrechern und Scheiterern gehörten. Die Zufriedenheit der Jugendhilfe mit dem Programm war hoch. Auf diese Weise wurden den entsprechenden Kindern Erziehung und Therapie in ihrem gewohnten Setting ermöglicht.

\section{Schluss}

Da ADHS oder die nach ICD-10 enger gefassten hyperkinetischen Störungen zu den häufigsten kinder- und jugendpsychiatrischen Erkrankungen gehören, ist naturgemäß die Schnittstelle zur Jugendhilfe groß. Sicherlich kann eine nicht geringe Zahl von Kindern mit unkomplizierter Aufmerksamkeitsstörung, auch ohne Jugendhilfemaßnahmen versorgt werden, wobei im Einzelfall die evtl. resultierende Teilhabebeeinträchtigung eben vom sozialen Umfeld, vom Milieu abhängt und auch hier nicht kategorisch von der Diagnose auf die Teilhabebeeinträchtigung bzw. die nicht vorhandene Teilhabebeeinträchtigung bei einer alleinigen Aufmerksamkeitsstörung geschlossen werden kann.

Häufig unterversorgt sind Kinder mit Aufmerksamkeitsstörungen in Jugendhilfemaßnahmen, insbesondere in stationären Hilfen. Sie haben meistens eine ungünstige Prognose, weil sich bei innen, neben der Aufmerksamkeitsstörung auch eine Störung des Sozialverhaltens herausgebildet hat. Dies betrifft mehr als $1 / 5$ der Heimkinder und diese benötigen dringend eine adäquate Behandlung, um weitere Verschlechterungen ihrer Situation und weitere Beziehungsabbrüche zu verhindern. Ausländische Kollegen sind nach wie vor sehr erstaunt über die hohe Zahl von Kindern mit Aufmerksamkeitsdefizitsyndromen und Störungen des Sozialverhaltens, welche wir in Deutschland stationär behandeln (vgl. Stang 2007). Derzeit werden in Westdeutschland 8,7 Jungen auf 100.000 Einwohner, in Ostdeutschland 25,3 Jungen auf 100.000 Einwohner meist mit hyperkinetischen Störungen mit Störungen des Sozialverhaltens (F 90.1) hospitalisiert. Die Dauer der Hospitalisierung beträgt im Osten im Mittel 38 Tage, im Westen 42 Tage. Auch diese Zahlen machen die große Schnittmenge zwischen schwerstauffälligen Kindern mit hyperkinetischer Störung des Sozialverhaltens mit ungünstiger Prognose zwischen Jugendhilfe und Jugendpsychiatrie deutlich. Hier sind nach wie vor Drehtüreffekte, verbunden mit ständigen Einrichtungswechseln zu beobachten. Unser vom BMBF und den Krankenkassen gefördertes Modell- 
projekt konnte gerade für dieses Klientel aufzeigen, dass hier Hospitalisierungen fast um die Hälfte reduziert werden konnten und damit zum Wohle der Betroffenen, aber auch zum Wohle der Versichertengemeinschaft, eine kontinuierlichere Betreuung vor Ort durch eine adäquate kinderpsychiatrische Versorgung in Kinderheimen erzielt werden konnte. Es wäre dringend zu wünschen, dass sich die fachpolitische Debatte weniger auf mittelschichtwendige Einzelfälle der befürchteten Leistungserschleichung über das KJHG konzentriert, sondern dass man sich wirklich um die schwer betroffenen Kinder mit hyperkinetischer Störung des Sozialverhaltens in beiden Systemen kompetenter und in abgestimmterer Weise kümmert.

\section{Literatur}

Bellis, D. (2001). Development traumatology: The psychobiological development of maltreated children and its implications for research, treatment, and policy. Development and Psychopathology 13(3): 539-564.

Fegert, J. M. (2004a). Behinderung - Versuch einer Begriffsbestimmung. Handbuch Jugendhilfe- Jugendpsychiatrie. J. M. Fegert and C. Schrapper. Weinheim und München, Juventa: 185-201.

Fegert, J. M. (2004 b). Der Beitrag der Kinder- und Jugendpsychiatrie zur Feststellung seelischer Behinderung und drohender seelischer Behinderung. Handbuch Jugendhilfe- Jugendpsychiatrie. J. M. Fegert and C. Schrapper. Weinheim und München, Juventa: 209-222.

Fegert, I. M., Glaeske, G. et al. (2002). Arzneimittel-Versorgung von Kindern mit hyperkinetischen Störungen anhand von Leistungsdaten der GKV. Projektbericht Kooperationsprojekt Universität Bremen, Zentrum für Public Health - Universitätsklinikum Ulm, Klinik für Kinder- und Jugendpsychiatrie/Psychotherapie.

Fegert, J. M., Roosen-Runge, G. et al. (2008). Stellungnahme zur Eingliederungshilfe nach $§ 35$ a SGB VIII der Kommission Jugendhilfe der kinder- und jugendpsychiatrischen Fachgesellschaften. Das Jugendamt 81 (4): 177-186.

Fegert, J. M., Schrapper, C. (2004). Handbuch Jugendhilfe - Jugendpsychiatrie. Interdisziplinäre Kooperation. München, Juventa Verlag Weinheim und München.

Hässler, F., Fegert J. M. (2004). Hyperkinetische Störungen. Psychiatrie und Psychotherapie des Kindes- und Jugendalters. C. Eggers, J. M. Fegert and F. Resch. Heidelberg, Springer: 819-848.

Meltzer, H., Lader, D. et al. (2003). The mental health of young people looked after by local authorities in Scotland: summary report.

Ravens-Sieberer, U. (2006). Modul Psychische Gesundheit. Bella-Studie. Berlin, Robert-Koch-Institut: www. kiggs.de.

Santosh, P. J., Taylor, E. et al. (2005). Refining the diagnoses of inattention and overactivity syndromes: A reanalysis of the multimodal Treatment study of attention deficit hyperactivity disorder (ADHD) based on ICD-10 criteria for hyperkinetic disorder. Clinical Neuroscience Research (5): 307-314.

Schmid, M., Goldbeck, L. et al. (2008). Prevalence of mental disorders among adolescents in German youth welfar institutions. Child and Adolescent Psychiatry and Mental Health: www.capmh.com.

Schrapper, C. (2004). Teilhabe ermöglichen. Sozialpädagogische Konzepte für die Aufgaben der Jugendhilfe bei Hilfen nach $\oint 35$ a KJHG. Handbuch Jugendhilfe - Jugendpsychiatrie. Interdisziplinäre Kooperation. J. M. Fegert and C. Schrapper. München, Juventa Verlag Weinheim und München: 203-207.

Stang, A. (2007) Hospitalisierungsraten von Kindern mit hyperkinetischen Störungen in Deutschland. Deutsches Ärzteblatt, 104 (19): 1306-1311.

Wiesner, R. (2006). SGB VIII, Kinder- und Jugendhilfe. Berlin, Beck. 